\title{
Supernova 1604, Kepler's supernova, and its remnant
}

\author{
Jacco Vink \\ University of Amsterdam, Science Park 904, 1098 XH Amsterdam \\ j.vink@uva.nl
}

\begin{abstract}
Supernova 1604 is the last Galactic supernova for which historical records exist. Johannes Kepler's name is attached to it, as he published a detailed account of the observations made by himself and European colleagues. Supernova 1604 was very likely a Type Ia supernova, which exploded 350 pc to $750 \mathrm{pc}$ above the Galactic plane. Its supernova remnant, known as Kepler's supernova remnant, shows clear evidence for interaction with nitrogen-rich material in the north/northwest part of the remnant, which, given the height above the Galactic plane, must find its origin in mass loss from the supernova progenitor system. The combination of a Type Ia supernova and the presence of circumstellar material makes Kepler's supernova remnant a unique object to study the origin of Type Ia supernovae. The evidence suggests that the progenitor binary system of supernova 1604 consisted of a carbon-oxygen white dwarf and an evolved companion star, which most likely was in the (post) asymptotic giant branch of its evolution. A problem with this scenario is that the companion star must have survived the explosion, but no trace of its existence has yet been found, despite a deep search.
\end{abstract}

\section{Contents}

\section{Introduction}

2 The supernova remnant, its distance and its multiwavelength properties

2.1 Position, distance estimates and SN 1604 as a runaway system . . 3

2.2 X-ray imaging spectroscopy and SN 1604 as a Type Ia supernova

2.3 The circumstellar medium as studied in the optical and infrared . . 9

3 The dynamics of Kepler's SNR

3.1 Velocity measurements . . . . . . . . . . . . . . . . 10

3.2 Hydrodynamical simulations of Kepler's SNR . . . . . . . . . 12 
4 The progenitor system of SN 1604

4.1 Elevated circumstellar nitrogen abundances, silicates and a single degenerate scenario for SN $1604 \ldots \ldots$. . . . . . . . . . 14

4.2 Problems with a single degenerate Type Ia scenario for SN 1604 . 15

4.3 Was SN 1604 a core-degenerate Type Ia explosion? . . . . . . . . 15

4.4 What can we learn from the historical light curve of SN 1604? . . 16

5 Conclusions

\section{Introduction}

Supernova (SN) 1604 is the last of the historical supernovae. We know of at least two other supernovae with later explosion dates, those associated with the supernova remnants Cassiopeia A and G1.9+0.3 (Green et al, 2008), but no known historical records of the associated supernova events exist.

The discovery of SN 1604 occurred on October 9, 1604 (Gregorian calendar), early in the evening when all interested in astronomy gazed the sky to watch the conjunction of Mars, Jupiter and Saturn. This special circumstance probably helped the early discovery of the supernova at around 20 days before maximum brightness (Stephenson and Green, 2002). The occurrence of a new star in conjunction with three bright planets lead to fierce debates among contemporary astronomers, most of whom still held partial medieval world views, with many elements of astrology. Of all these astronomers, it is in particular Johannes Kepler's name that is now attached to this supernova, because he wrote a book "De Stella Nova in Pede Serpentarii" ("On the new star in the foot of the Snake [Ophichius]") about the new star, in which he published his own observations of the supernova and those by various European colleagues, and discusses the importance of the new star, including a possible link to the star of Bethlehem. Kepler only started observing SN 1604 relatively late, October 17, due to unfortunate weather circumstances in Prague, his city of residence. Kepler and his contemporaries attached a great deal of metaphysical significance to the appearance of the new star: "The star's significance is a difficult matter to establish and we can be sure of only one thing: that either the star signifies nothing at all for Mankind or it signifies something of such exalted importance that it is beyond the grasp and understanding of any man". Kepler himself, in fact, was a bit more cautious about the metaphysical implications of the event than some of his contemporaries (Granada, 2005).

Currently, the remnant of SN 1604, labeled G4.5+6.8, but usually called Kepler's supernova remnant (SNR), or even "Kepler" for short, is one of the best studied SNRs. It appears that Kepler's SNR has indeed a wide ranging significance, but not for metaphysical reasons, but for understanding the nature of Type Ia supernovae. SN 1604 was almost certainly a Type Ia supernova, but, peculiarly, the SNR itself is interacting with a nitrogen-rich circumstellar medium (CSM) at a distance of 2-3 pc from the explosion centre, suggesting that the progenitor system 
had significant mass loss.

As described in more detail in Sections 3 and 6, two basic types of supernova Type Ia progenitor models exists (Maoz et al, 2014): 1) the single degenerate model, according to which a $\mathrm{CO}$ white dwarf accretes from an (evolved) stellar companion until the pressure in the core leads to the thermonuclear explosion of the white dwarf, at the moment that the progenitor has a mass approaching the Chandrasekar mass $\left(1.38 \mathrm{M}_{\odot}\right)$, and 2) the double degenerate model, according to which Type Ia explosions are triggered by the merging of two white dwarfs. The time scale from birth of the binary system to the merging of the two white dwarfs is generally expected to be a few $10^{9} \mathrm{yr}$ (Maoz et al, 2014), since the two stars are brought closer to each other due gravitational radiation. The fact that Kepler's SNR is interacting with CSM is, therefore, more consistent with the single degenerate scenario. However, as will be discussed, this scenario is also not fully consistent with the properties of Kepler's SNR, in particular with the lack of a surviving donor star.

\section{The supernova remnant, its distance and its multiwave- length properties}

\subsection{Position, distance estimates and SN 1604 as a runaway system}

Kepler's SNR was discovered by Walter Baade as "a small patch of nebulosity close to the expected place" (Baade, 1943). Minkowski(1959) revealed that the spectrum of the optical nebula displayed strong [NII] emission as compared to $\mathrm{H} \alpha$ emission. The $\mathrm{H} \alpha /[\mathrm{NII}]$ is concentrated toward the north/northwestern region of the SNR and a bar-shape region across the center, roughly oriented in the NW-SE direction (Fig. 11). The overall extent of the SNR is more clear from radio and X-ray images of the SNR, revealing a roughly spherical shell with two protrusions ("Ears") in the NW and SE (e.g. Delaney and Rudnick, 2003; Reynolds et al, 2007). The SNR's center is located at $\alpha_{\mathrm{J} 2000}=17 \mathrm{~h} 30 \mathrm{~m} 41 \mathrm{~s}, \delta_{\mathrm{J} 2000}=-21^{\circ} 29^{\prime} 32^{\prime \prime}$, in Galactic coordinates $l=4.52^{\circ}, b=6.82^{\circ}$. The angular radius of the SNR is approximately $1.8^{\prime}$ (not taking into account the "Ears").

The distance to the SNR is uncertain, with estimates varying from $d=3.2 \mathrm{kpc}$ (Danziger and Goss, 1980) to $d=12 \mathrm{kpc}$ (van den Bergh et al, 1973). The long distance was based on the maximum visual magnitude of the supernova event of $V_{\max } \approx-2.5$, as estimated by Baade (1943) from the historical records. After correcting for absorption $\left(A_{\mathrm{V}}=2.17-3.47\right.$, van den Bergh and Kamper, 1977; Danziger and Goss, 1980), the peak magnitude can then be compared to the absolute magnitude of Type Ia supernovae of $M_{\mathrm{V}}=-19.0 \pm 0.5$. A Hubble parameter was adopted of $H_{0}=100 \mathrm{~km} / \mathrm{s} / \mathrm{Mpc}$.

More recent distance estimates, not based on the historical peak brightness of the supernova, vary widely, ranging from $d=3.9 \pm 1.4 \mathrm{kpc}$ (Sankrit et al, 2005) based on combining proper motion and Doppler broadening of $\mathrm{H} \alpha$ emission, up to, 


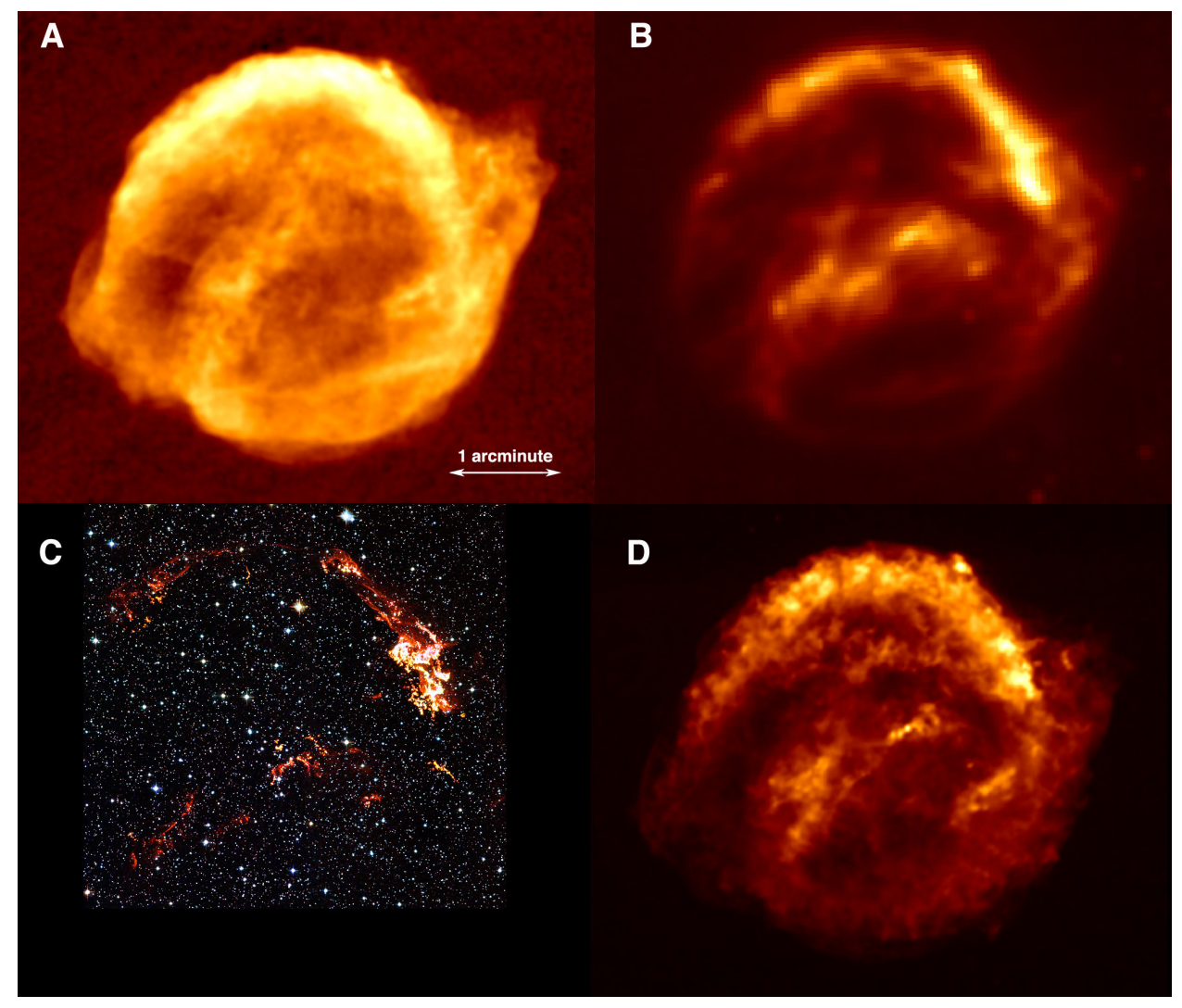

Figure 1: Kepler's SNR at different wavelengths: a) Radio VLA (4.85 GHz) map (Delaney and Rudnick, 2003); b) $24 \mu \mathrm{m}$ dust emission as observed by the Spitzer MIPS instrument (Blair et al, 2007); c) optical image (H $\alpha$, [NII] and [OIII]) obtained by the Hubble Space Telescope/ACS instrument (credit: ESA/NASA, The Hubble Heritage Team (STScI/AURA)); d) Chandra X-ray image in the iron-rich 0.7-1.0 keV band (Reynolds et al, 2007). Intensity scaling of Fig. a, b, and d are square-root scalings, bringing out fainter details. 
or even beyond, 6-7 kpc based on the angular size of the SNR, combined with the energetics of the supernova (Aharonian et al, 2008, Chiotellis et al, 2012; Patnaude et al, 2012, see Section 3 for details). Radio absorption measurements toward the SNR are consistent with a distance in the range 4.8-6.4 kpc (Reynoso and Goss, 1999).

The Galactic latitude of Kepler's SNR $\left(b=6.8^{\circ}\right)$ implies a large height above the Galactic plane of $z=594 d_{5}$ pc (with $d_{5}$ the distance in units of $5 \mathrm{kpc}$ ). The large separation from active star forming regions suggests that SN 1604 was Type Ia supernova, in agreement with the reconstructed supernova light-curve, as already pointed out by Baade (1943). However, the large amounts of nitrogen present in the optical nebula can only have come from material processed by the CNO nucleosynthesis cycle, suggesting a stellar wind origin for the optical nebula. This lead Bandiera (1987) to suggest that the progenitor was a massive star, which escaped the Galactic plane with a high velocity. The high proper motion of the progenitor is in agreement with observations of the kinematics of the optical nebula (Section 3 ). Given the historical light curve, a Type II origin of SN 1604 was excluded, but a Type Ib supernova was consistent with both the light curve and the idea of a massive runaway star as the progenitor of the supernova Bandiera (1987).

\subsection{X-ray imaging spectroscopy and SN 1604 as a Type Ia supernova}

The idea of a Type Ib supernova origin for SN 1604 was discredited with the advent of X-ray imaging spectroscopy with CCDs. These type of detectors were first used by ASCA, and it provides now still the most often used instrumentation for X-ray observatories such as Chandra, XMM-Newton, and Suzaku. The X-ray spectrum of Kepler's SNR (Fig. 3) shows that the dominant line emission is caused by Fe-L transitions (Kinugasa and Tsunemi, 1999, Cassam-Chenaï et al, 2004; Reynolds et al, 2007), which is very indicative of Type Ia SNRs, since they produce 0.3-1.3 $\mathrm{M}_{\odot}$ of $\mathrm{Fe}$ and $\mathrm{Fe}$-group elements. In addition line emission from intermediate mass elements like silicon, sulphur, argon and calcium, are prominent. Unlike Kepler's SNR, young core collapse SNRs on the other hand, show dominant emission from oxygen, neon and magnesium. Recently, Katsuda et al (2015) confirmed the high nitrogen abundance in Kepler's SNR using high resolution X-ray spectroscopy with the XMM-Newton reflective grating spectrometer.

The spatial distribution of the X-ray line emission indicates that the ejecta are layered according to element mass, with the intermediate mass elements located, on average, at a larger radius than iron (Cassam-Chenaï et al, 2004, illustrated in Fig. 2). This distribution is similar to that of other Type Ia SNRs like Tycho's SNR (SN 1572, Hwang and Gotthelf, 1997) and SNR 0519-69.0 (Kosenko et al, 2010), but Fe-L emission is more prominent in Kepler's than in Tycho's SNR, whereas SNR 0519-69.0 and SN 1006 display more oxygen line emission from the outer layers.The relatively high iron abundance in Kepler's SNR, as compared to other Type Ia SNRs, suggests that SN 1604 was an iron/nickel-rich supernova, similar to the peculiar Type Ia supernova SN1991T (Patnaude et al, 2012, but see 


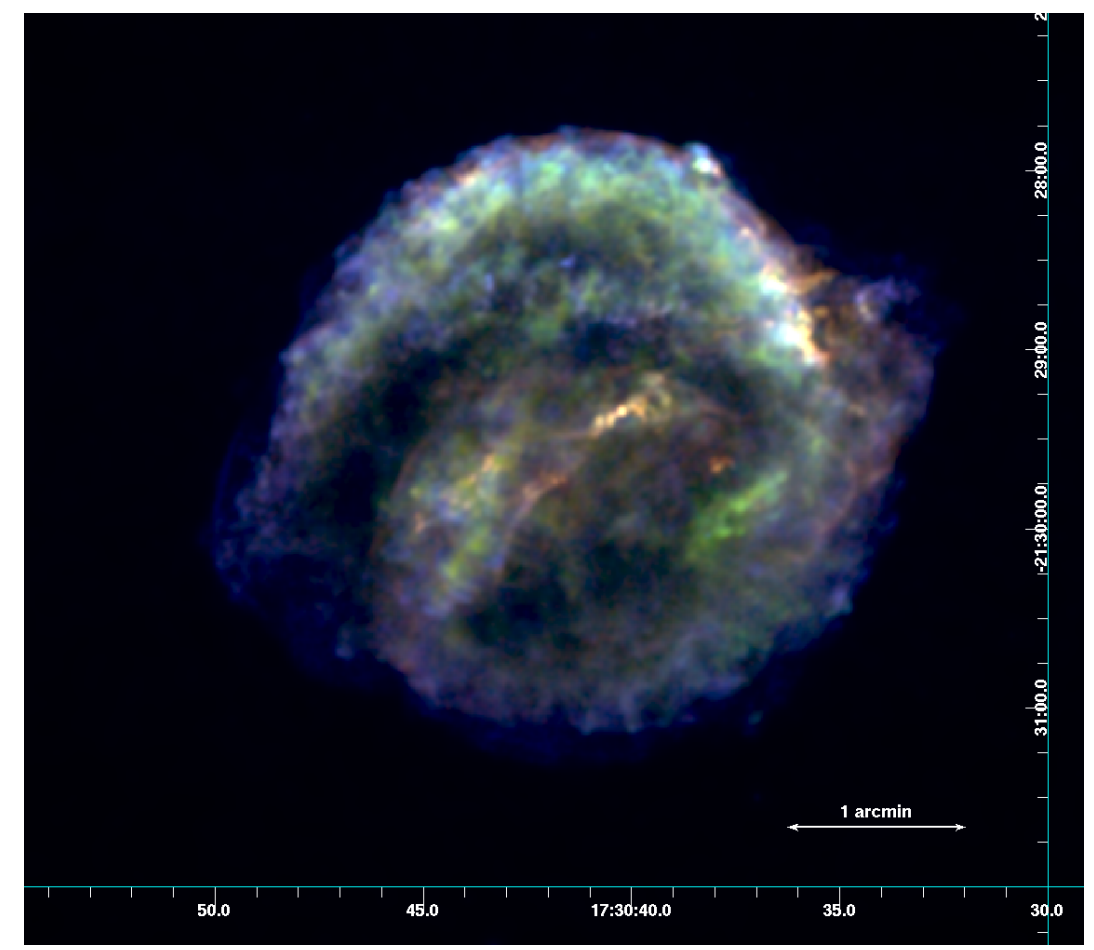

Figure 2: Chandra X-ray image, with the three image channels (red, green, blue) corresponding to oxygen emission (0.5-0.7 keV), Fe-L emission (0.7-1 keV) and $\mathrm{Si}-\mathrm{K}$ emission (1.7-1.9 keV), respectively. It can be seen that the $\mathrm{Si}-\mathrm{K}$ emission (blue) peaks more outward than the the Fe-L shell emission (green). Peaks in the oxygen emission (red/yellowish) occur at the outskirts in the northeast and along the bar, suggesting that the emission comes from shock-heated, circumstellar material. 


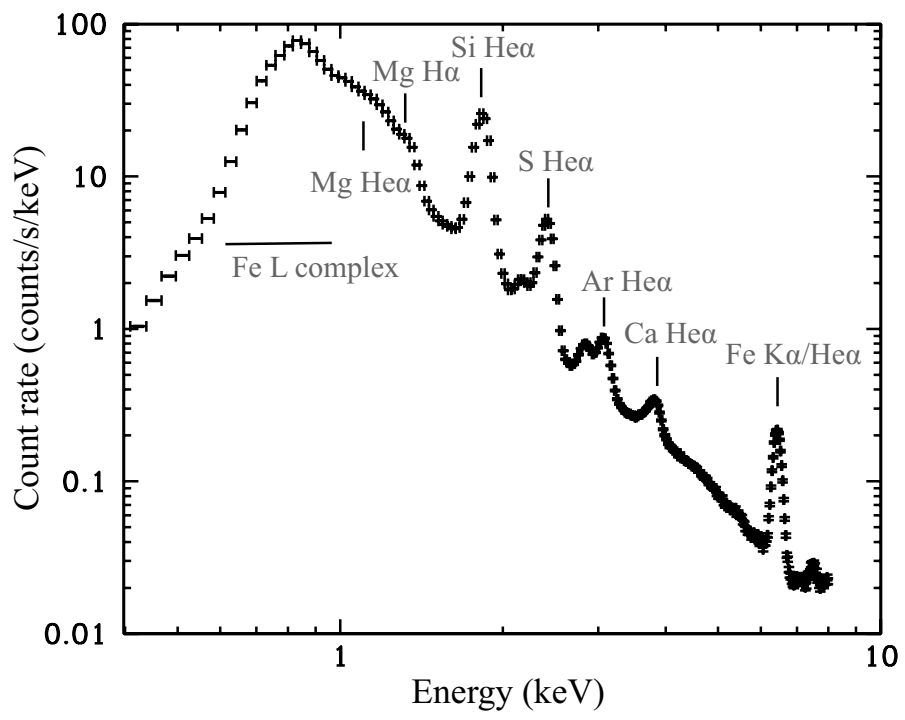

Figure 3: The Chandra ACIS-S X-ray spectrum of Kepler's SNR, observed in 2006. The "bump" between 0.7- $1.2 \mathrm{keV}$ consists mainly of Fe-L emission, consisting of complex of lines from Fe XVII to Fe XXIV. The prominence of this Fe-L complex in the spectrum of Kepler's SNR is indicative of a Type Ia origin.

Section 4.47.

Recently it has become possible to detect line emission from the uneven elements manganese $(\mathrm{Mn})$ and chromium $(\mathrm{Cr})$. The $\mathrm{Mn}$ yields for supernovae are a result of the presence of neutron-rich nuclei in the progenitors, which ultimately is linked to the nitrogen abundance of the progenitor at the main sequence. Hence, it can be used to infer the metallicity of the progenitor. Park et al (2013) used the $\mathrm{Mn} / \mathrm{Cr}$ ratio to show that SN 1604 must originate from a star with super-solar metallicity, ruling out a halo star progenitor.

The continuum X-ray emission of Kepler's SNR, best distinguished in the 4-6 $\mathrm{keV}$ band (blue in Fig. 4), shows narrow filaments tracing the outer region of the SNR. Most of this emission, especially the narrow filaments in the SE, are thought to be caused by X-ray synchrotron emission from 10-100 TeV shock accelerated electrons (Helder et al, 2012, for a review). The widths of the filaments can be used to estimate the post-shock magnetic fields, indicating for Kepler's SNR a magnetic field strength of $B \approx 120 \mu \mathrm{G}$. Since the $10-100 \mathrm{TeV}$ lose their energy very fast in such a magnetic field $(\sim 10-20 \mathrm{yr})$, the $\mathrm{X}$-ray synchrotron radiation is an excellent tracer of the shock front. Some of the X-ray synchrotron filaments are situated along the edges of the central bar (Fig. 4), suggesting that we observe the shock front under a (nearly) edge-on viewing angle. Given the position of the bar in the center of the SNR, this viewing angle indicates a peculiar, perhaps halter-like, morphology of the SNR in three dimensions (Burkey et al, 2013). 


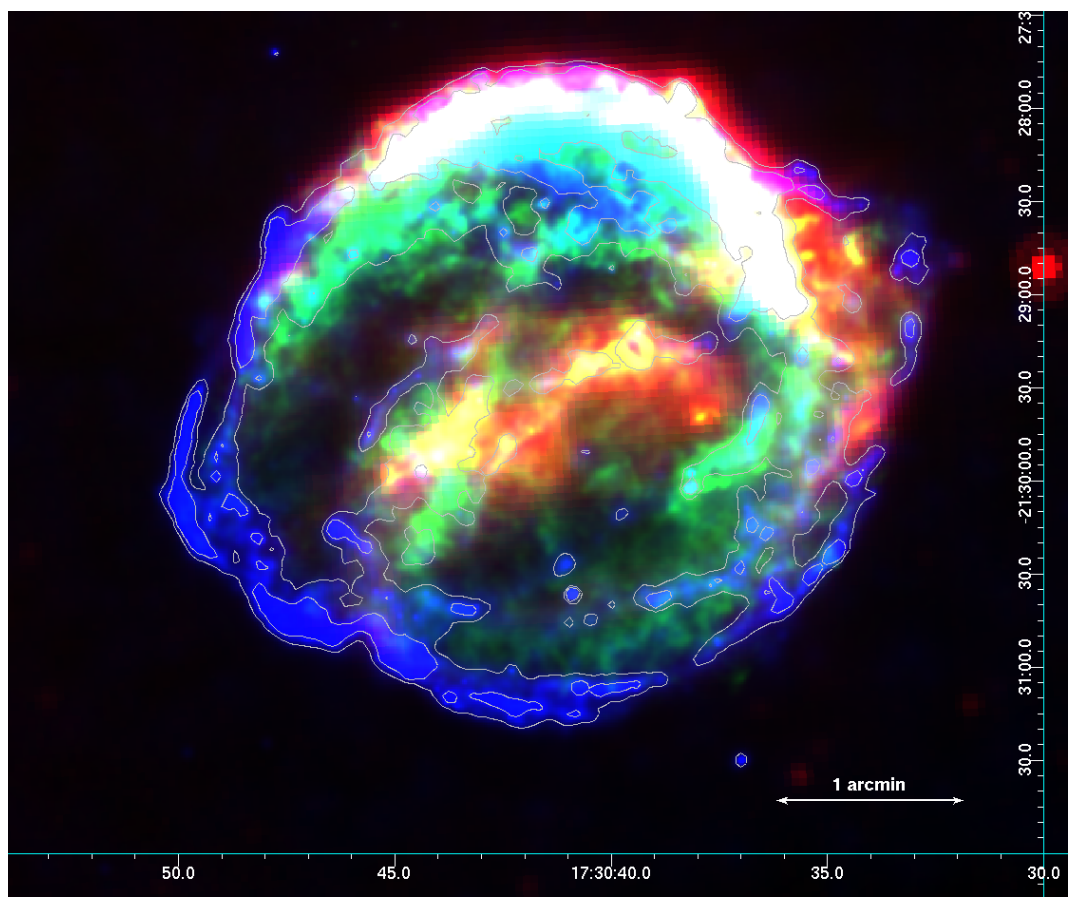

Figure 4: Multiwavelength image of Kepler's SNR. The red channel represents the $24 \mu \mathrm{m}$ dust emission as observed by the Spitzer MIPS instrument (Blair et al, 2007). The green channel is the Si-K band (1.7-2.0 keV), representing the bulk of the thermal X-ray emission, associated with Si-rich ejecta. The Si-K emission comes on average from a smaller radius than the dust emission, indicating that the dust originates from the swept up circumstellar medium, rather than the ejecta. The blue channel (enhanced with contours) shows the X-ray continuum emission in the 4-6 keV band. In the SE the emission is dominated by synchrotron emission from close to the shock front. In the NW it is probably a mixture of synchrotron emission and thermal bremsstrahlung. The X-ray bands are taken from the 2006 Chandra observations of Kepler's SNR (Reynolds et al, 2007). 


\subsection{The circumstellar medium as studied in the optical and infrared}

The shocked interstellar medium in Kepler's SNR is best studied in the optical and infrared. The optical emission, dominated by hydrogen line emission, comes from northwestern part of the SNR and from the central bar. The optical emission can be divided in emission caused by either radiative or non-radiative shocks (Dennefeld, 1982; Fesen et al, 1987; Blair et al, 1991; Sankrit et al, 2008). Radiative shock emission is characterised by a mix of hydrogen line emission (Balmer and Lyman lines) and forbidden line emission from NII, OIII, and SII. The $\mathrm{H} \alpha$ line emission is in those cases double peaked, since NII has among others line emission at $\lambda=$ $654.9 \mathrm{~nm}$, close to $\mathrm{H} \alpha(\lambda=656.4 \mathrm{~nm})$. Forbidden line emission comes from regions heated by slow shocks $\left(\lesssim 200 \mathrm{~km} \mathrm{~s}^{-1}\right.$ ), which heat plasma to temperatures below $\sim 10^{6} \mathrm{~K}$. For this temperature regime radiative losses by optical/UV line emission causes rapid cooling. For Kepler's SNR the overall shock speed is several thousand $\mathrm{km} \mathrm{s}^{-1}$. So the radiative emission must come from dense knots $(n \gtrsim$ $10^{3} \mathrm{~cm}^{-3}$ Leibowitz and Danziger, 1983), in which locally the shock wave has decelerated considerably. The [NII]/hydrogen line ratios measured for radiative shocks in Kepler's SNR suggests an overabundance in nitrogen of a factor 2-3.5 (Leibowitz and Danziger, 1983; , Blair et al, 1991).

The non-radiative emission is caused by neutral hydrogen atoms entering the shock, after which they may not be immediately ionised, but first get into an excited state, or undergo will charge exchange with shock-heated protons behind the shock front. $\mathrm{H} \alpha$ line spectra of non-radiative shocks typically consist of narrowline emission caused by direct excitation of the neutrals, and broad-line emission caused by hot protons that just picked up an electron through charge exchange. The narrow line width and line center can be used to measure the plasma speed and temperature of the un-shocked gas, whereas the broad-line width can be used to measure the temperature immediately $\left(\lesssim 10^{15} / n_{\mathrm{p}} \mathrm{cm}\right)$ behind the shock (see Section 3). The $\mathrm{H} \alpha$ flux of the non-radiative filaments can be used to infer densities as well, which appears to be of the order of $\sim 10 \mathrm{~cm}^{-3}$ (Blair et al, 1991). This is surprisingly large, given the height above the Galactic plane of $594 d_{5} \mathrm{pc}$. The optical emission from Kepler's SNR, therefore indicates that the SNR is interacting with dense, CNO processed material, which, given the isolated nature of the SNR high above the Galactic plane, can only be understood by assuming that the circumstellar material originates from the progenitor system of SN 1604.

In the infrared Kepler's SNR has been observed in the wavelength range from $12-100 \mu \mathrm{m}$ by all major infrared observatories (IRAS, ISO, Spitzer, Herschel, see Braun, 1987; Douvion et al, 2001; Blair et al, 2007; Gomez et al, 2012; Williams et al, 2012). Additional emission for wavelength larger than $100 \mu \mathrm{m}$, even up to $850 \mu \mathrm{m}$ may be present (Morgan et al, 2003; Gomez et al, 2012), but at long wavelengths background subtraction is difficult, and even at $100 \mu \mathrm{m}$ there is already a factor three discrepancy between IRAS and Herschel based flux measurements (Gomez et al, 2009).

Infrared emission from SNRs is caused by warm dust, heated by collisions with 
hot electrons and ions. The dust temperature is established by the equilibrium between collisional heating and thermal emission (Draine, 1981; Dwek, 1987). Both heating and emission depend on the surface area of the dust grains, and for large dust grains the temperature is independent of the size of the dust particles. However, since the wavelength of the radiation and the dust particle size can be comparable, the dust emission cannot be adequately described by Stefan-Boltzmann's law. Moreover, the emission spectrum also depends on the composition of the dust.

Infrared photometry of Kepler's SNR indicates dust temperatures of 80-120 K (Braun, 1987; Douvion et al, 2001; Blair et al, 2007, Gomez et al, 2012; Williams et al, 2012), whereas more detailed spectra obtained with the ISO-SWS and SpitzerIRS spectrometers (respectively Douvion et al, 2001; Williams et al, 2012) indicate that the dust particles themselves are silicates (silicon oxides). Interestingly, the modelling of the spectra gives rise to different interpretations. Douvion et al (2001) conclude that the dust comes from regions with densities of $n_{\mathrm{e}}=500-7500 \mathrm{~cm}^{-3}$ with plasma temperatures of $(0.4-6) \times 10^{5} \mathrm{~K}$. On the other hand, Williams et al (2012) estimates the densities to be $n_{\mathrm{e}} \approx 5 \mathrm{~cm}^{-3}$ in the South to $n_{\mathrm{e}} \approx 50 \mathrm{~cm}^{-3}$ in the North, but assumes that the plasma temperatures are consistent with the $\mathrm{X}$-ray measured temperatures ( $T_{\mathrm{e}} \approx 10^{7} \mathrm{~K}$, and ion temperatures a factor ten higher). Both conditions may arise in Kepler's SNRs as the radiative shocks are going through very dense regions, whereas the non-radiative shocks are responsible for the $\mathrm{X}$ ray emitting plasma. Indeed, several of the regions analysed by Williams et al (2012) contain non-radiative shock emission. The infrared spectrum of the region in which radiative shock emission is dominant is characterised by bright forbidden line emission in the infrared (among others [Si II], [Fe II]). It is important to note that the location of the dust emission indicates that all the dust is associated with shocked CSM, and not with supernova ejecta. This appears to be a characteristic of Type Ia supernovae, which do not seem to produce dust in their ejecta (Williams et al, 2012). This is in contrast to a core collapse SNR like Cas A (Lagage et al, 1996) or SN 1987A, where dust clearly is formed from supernova ejecta.

\section{The dynamics of Kepler's SNR}

\subsection{Velocity measurements}

The earliest measurements of velocities in Kepler SNR's were radial velocity measurements of the bright optical knots, indicating line emission that is blue shifted by about $230 \mathrm{~km} \mathrm{~s}^{-1}$ (Minkowski, 1959). Optical proper motion studies (van den Bergh and Kamper, 1977; Bandiera and van den Bergh, 1991) also indicated velocity offsets, indicating that the bright optical knots have a bulk velocity of 212 $d_{5} \mathrm{~km} \mathrm{~s}^{-1}$ in northwestern direction, away from the Galactic plane, whereas the expansion velocity, based on spherical expansion directed away from the centre of the SNR, is only $(74 \pm 29) d_{5} \mathrm{~km} \mathrm{~s}^{-1}$ (Bandiera and van den Bergh, 1991). The slow expansion velocity, corresponding to an expansion time scale of $32000 \mathrm{yr}$, indicates that the velocity of the optical knots reflects mostly the velocity of these 

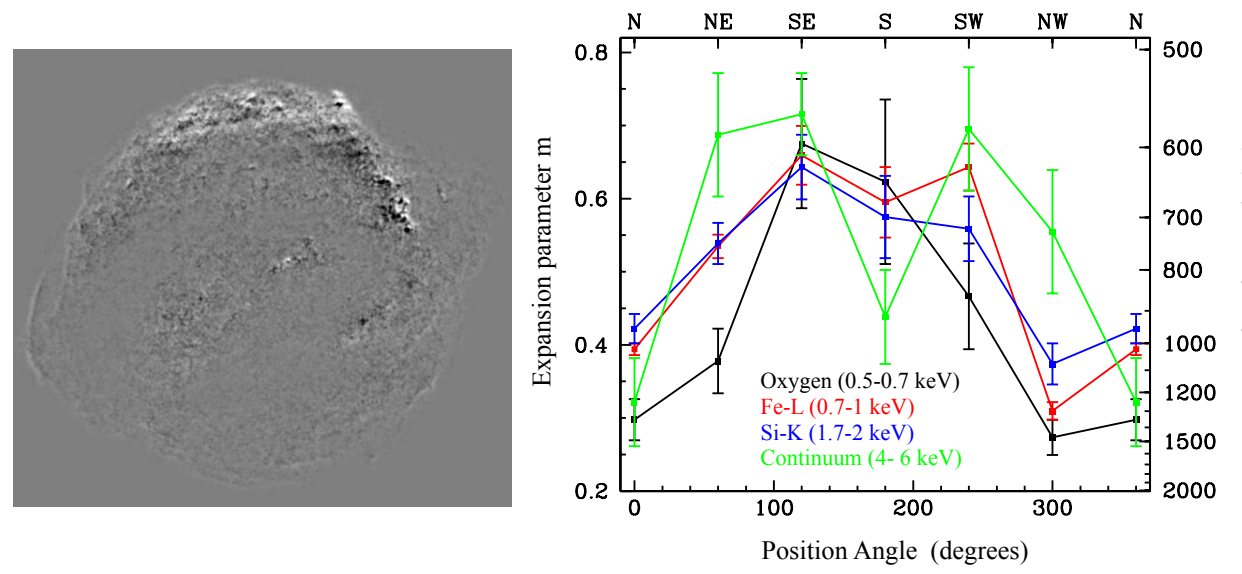

Figure 5: Left: Difference image between images in the 1-1.5 keV band in 2000 and 2006, as observed by Chandra. The shadow-like features are caused by proper motions. Right: X-ray expansion measurements based on these two Chandra images in various energy bands (adapted from figure in Vink, 2008).

knots prior to interaction with the SNR blast wave.

The more diffuse non-radiative $\mathrm{H} \alpha$ emission (Fesen et al, 1987) provides information on the local shock wave velocities through the width of the broad $\mathrm{H} \alpha$ component; and the narrow line component provides information on the velocity of the unshocked, partial neutral gas, immediately ahead of the shock. The latter informs us that the unshocked gas is blue shifted with about $180 \mathrm{~km} \mathrm{~s}^{-1}$ (Blair et al, 1991; Sollerman et al, 2003).

The width of the broad line $\mathrm{H} \alpha$ component is caused by thermal Doppler broadening of shock-heated protons. The reported $\mathrm{H} \alpha$ line FWHM of $\sim 1800 \mathrm{~km} \mathrm{~s}^{-1}$ translates into shock velocities of $1500-2000 \mathrm{~km} \mathrm{~s}^{-1}$ (Blair et al, 1991). Part of the uncertainty is caused by the uncertainty in electron-ion temperature ratio. Sankrit et al (2005) combined this shock velocity measurement with a measure proper motion of diffuse filament in the NW of $1.45 \pm 0.03$ "/16.33 yr to infer a distance to Kepler's SNR of 3.0-5.0 kpc. However, note that some of the Doppler broadening widths measured by Blair et al (1991) indicate shock velocities larger than $2000 \mathrm{~km} \mathrm{~s}^{-1}$. Notably, the filament labeled "NW diffuse emission" has a width of $3409 \pm 454 \mathrm{~km} \mathrm{~s}^{-1}$, which corresponds to a shock velocity range of 2300$4000 \mathrm{~km} \mathrm{~s}^{-1}$ (van Adelsberg et al, 2008), or even larger if cosmic-ray acceleration absorbs part of the shock energy-flux into the shock, which results in lower than expected post-shock temperatures (e.g. Vink et al, 2010).

The optical velocity measurements pertain to the densest regions of Kepler's SNR. The overall expansion of Kepler's SNR has been measured in the radio (Dickel et al, 1988, with VLA) and X-rays (Vink, 2008, Katsuda et al, 2008, see Fig. (5) using proper motion measurements. These measurements show that the northwestern part is expanding at a slower rate than the southwestern part. A use- 
ful way to characterise the expansion rate is in terms of the expansion parameter

$$
m \equiv \frac{R / t}{V},
$$

with $R$ the radius, $t$ the age of the SNR and $V$ the velocity. For proper motions both $R$ and $V$ depend in a similar way on the distance, so one can replace $R$ and $V$ with angular radius and proper motion. One can also calculate the expansion age $\tau_{\text {exp }} \equiv R / V$, in which case $m=t / \tau_{\exp }$, with $t$ the true age of the SNR. For SNRs in the Sedov-Taylor phase of the evolution we expect $R \propto t^{2 / 5}$ and hence $m=2 / 5$, whereas in the ejecta dominated phase one expects $m=0.7-0.9$ (Chevalier. 1982). It turns out that for Kepler's SNR in the Northwest $m=0.3-0.35$, whereas in the Southwest the expansion is faster, with $m=0.6-0.7$. The expansion parameter in the northwest is lower than $m=2 / 5$, which indicates that the shock must have encountered a density enhancement. Vink (2008) estimates that the total excess mass toward the northwestern region is about $1 \mathrm{M}_{\odot}$.

The proper measured proper motions translate in shock velocities of

$$
V_{\mathrm{sh}}=m 9920(\theta / 2.79) d_{5} \mathrm{~km} \mathrm{~s}^{-1}
$$

for an age of $400 \mathrm{yr}$, with $\theta=2.79^{\prime}$ the angular radius of Kepler's SNR. Given the measured values of $m$ the shock velocities around Kepler's SNR are, therefore, in the range of $2900-7000 d_{5} \mathrm{~km} \mathrm{~s}^{-1}$.

\subsection{Hydrodynamical simulations of Kepler's SNR}

As discussed above the density enhancements in the north-northwestern regions, as well as the evidence for high density, nitrogen-rich material suggests that the progenitor system of SN 1604 suffered significant mass loss. Borkowski et al (1994) simulated this situation in the context of a Type Ib origin of SN 1604 and Chiotellis et al (2012) adapted it for the case of a Type Ia scenario, which I will briefly describe here.

The basic idea is that a dense wind has emanated from the secondary star with a mass-loss rate of $\dot{M} \mathrm{M}_{\odot} \mathrm{yr}^{-1}$, and a wind velocity $v_{\mathrm{w}}$. The wind must have lasted long enough for the wind material to reach the current radius of Kepler's SNR $\left(2.6 d_{5} \mathrm{pc}\right)$ implying that the wind must have prolonged for at least $t_{\mathrm{w}} \gtrsim 2.5 \times$ $10^{5} d_{5}\left(v_{\mathrm{w}} / 10 \mathrm{~km} \mathrm{~s}^{-1}\right) \mathrm{yr}$. The total mass lost by the system is given by

$$
M_{\text {wind }} \gtrsim \dot{M} t_{\mathrm{w}}=\dot{M} \frac{R}{v_{\mathrm{w}}}=2.5\left(\frac{\dot{M}}{10^{-5} \mathrm{M}_{\odot} \mathrm{yr}^{-1}}\right)\left(\frac{v_{\mathrm{w}}}{10 \mathrm{~km} \mathrm{~s}^{-1}}\right)^{-1} d_{5} \mathrm{M}_{\odot} .
$$

This means that the mass loss rate cannot have been much higher than $10^{-5} \mathrm{M}_{\odot} \mathrm{yr}^{-1}$, as the total mass loss of the donor could not exceed the mass of a viable donor star for a Type Ia progenitor, which must lie in the range of 3-6 $\mathrm{M}_{\odot}$.

The optical proper motions of the knots indicate a space of around $250-280 \mathrm{~km} \mathrm{~s}^{-1}$ (212 $d_{5} \mathrm{~km} \mathrm{~s}^{-1}$ in NW direction and $180 \mathrm{~km} / \mathrm{s}$ toward us). This likely corresponds 
to the space velocity of the progenitor system. The combination of a spherical wind and a space velocity gives rise to a bow shock around the system in the direction of the motion of the system. The density of the wind as a function of radius $r$ is given by

$\rho_{\mathrm{w}}(r)=\frac{\dot{M}}{4 \pi r^{2} v_{\mathrm{w}}}=7.8 \times 10^{-25}\left(\frac{\dot{M}}{10^{-5} \mathrm{M}_{\odot}}\right)\left(\frac{v_{\mathrm{w}}}{10 \mathrm{~km} \mathrm{~s}^{-1}}\right)^{-1}\left(\frac{R}{2.6 \mathrm{pc}}\right)^{-2} \mathrm{gcm}^{-3}$.

The densities in the Northwest appear to be around $50 \mathrm{~cm}^{-3}$ (Section 2.3). The density in the wind may be lower, as the gas encountered by the SNR shock may have been shocked by the wind termination shock, and later by the SNR shock. However, we see that the mass loss rate cannot have been substantially lower $10^{-5} \mathrm{M}_{\odot} \mathrm{yr}^{-1}$, as otherwise the wind densities would fall below the densities of the CSM component in Kepler's SNR.

The termination radius of the wind is now given by assuming equilibrium between the ram pressure of the wind $P_{\mathrm{w}}=\rho_{\mathrm{w}} v_{\mathrm{w}}^{2}$ and the ram pressure of the interstellar medium, $P_{\mathrm{ISM}}=\rho_{\mathrm{ISM}} v_{\text {prog }}^{2}$, with $v_{\text {prog }}$ the velocity of the progenitor system. Hence,

$R_{\mathrm{ts}}=1.9\left(\frac{\dot{M}}{10^{-5} \mathrm{M}_{\odot}}\right)^{1 / 2}\left(\frac{v_{\mathrm{w}}}{10 \mathrm{~km} \mathrm{~s}^{-1}}\right)^{1 / 2}\left(\frac{v_{\mathrm{prog}}}{250 \mathrm{~km} \mathrm{~s}^{-1}}\right)^{-1}\left(\frac{n_{\mathrm{ISM}}}{10^{-3} \mathrm{~cm}^{-3}}\right)^{-1 / 2} \mathrm{pc}$,

with $n_{\text {ISM }}=10^{-3}$ an estimate for the ISM at $500 \mathrm{pc}$ above the Galactic plane. This estimate for the termination shock radius is in reasonable agreement with the radius of Kepler's SNR.

The simulations of Chiotellis et al (2012) show that both the bow-shock like shape of Kepler's SNR as well as the measured expansion parameters can be reasonably well reproduced if SN 1604 indeed went off inside a stellar wind bubble with a systematic velocity of $250 \mathrm{~km} \mathrm{~s}^{-1}$. It was assumed that the ejecta mass was $1.4 \mathrm{M}_{\odot}$, typical for an exploding white dwarf. The simulations showed that for a distance of $4 \mathrm{kpc}$ the radius of the termination shock must be around $2 \mathrm{pc}$. However, a SNR with an explosion energy of $10^{51}$ erg going off inside such a smaller bubble, would have moved through the wind bubble and the shock would have penetrated into the ISM, which is in disagreement with the observations. One can reproduce the characteristic of Kepler's SNR at this distance, but only if the explosion energy is reduced to $2 \times 10^{50} \mathrm{erg}$. This is in disagreement with the typical Type Ia explosion energies of $1.2 \times 10^{51} \mathrm{erg}$ (Woosley et al, 2007). Moreover, the high Fe content of Kepler's SNR rather suggest that SN 1604 was a relatively energetic type Ia event (Park et al, 2013). This problem does not occur if Kepler's SNR has a distance of $\gtrsim 6 \mathrm{kpc}$.

A related hydrodynamical study by Patnaude et al (2012) concentrates on the impact of hydrodynamics on the resulting X-ray spectra. The wind bubble evolution itself is not modelled, but an $1 / r^{2}$ density distribution is assumed. They confirm that normal Type Ia explosion energies are not consistent with a distance 
of Kepler's SNR of $4 \mathrm{kpc}$, but that instead a distance of $7 \mathrm{kpc}$ should be considered. Toledo-Roy et al (2014) follow more closely the scenario of Chiotellis et al (2012), but the simulations are in 3D and the wind loss is assumed to be anisotropic. As a result more structure is found in the simulated X-ray maps, some of which may explain for example the central bar in Kepler's SNR (e.g. their Fig. 10). In general, the anisotropy of the wind can result in a peculiar structure. Burkey et al (2013) simulated the evolution of the SNR inside a wind strongly varying as a function of the polar axis. However, the simulation does not take into account the effects of the velocity of the system as a whole, and the expansion parameter of the plasma.

\section{The progenitor system of $\mathrm{SN} 1604$}

\subsection{Elevated circumstellar nitrogen abundances, silicates and a single degenerate scenario for $\mathrm{SN} 1604$}

Chiotellis et al (2012) argued that the mass loss properties inferred for the progenitor system of SN 1604 suggest that the progenitor system consisted of CO white dwarf accreting wind material from a Asymptotic Giant Branch (AGB) star. Indeed, the high mass loss rates and the wind expansion time scale are in good agreement with what is generally inferred for AGB stars. Given the relatively high total mass loss $\left(\sim 2.5 \mathrm{M}_{\odot}\right.$, plus matter that has accreted onto the white dwarf), it does mean that the donor star must have been a relatively massive star at the main sequence.

In addition, the nitrogen-rich abundance of the wind, and the silicate dust (Sect. 2.3 also gives clues about the donor star. The nitrogen-richness can be explained by hot bottom burning (e.g. Karakas, 2010), a process in which hydrogen is burned through CNO cycle at the base of the outer convective envelope. This brings nitrogen to the surface. Hot bottom burning occurs in stars with initial masses larger than 4-5 $\mathrm{M}_{\odot}$. Silicate dust predominantly forms in winds in which the carbon/oxygen ratio is smaller than 1 . Otherwise the oxygen binds to carbon, making $\mathrm{CO}$, leaving no oxygen for the build up of silicates. In this light it is interesting that McSaveney et al (2007) found two AGB stars in the Large Magellanic Clouds, which have elevated nitrogen abundance and a $C / O<1$. The masses of these two stars are inferred to be $4 \mathrm{M}_{\odot}$ and $6 \mathrm{M}_{\odot}$.

Taken all the evidence together we seem to have a rather consistent scenario for the progenitor system of SN 1604 and the evolution of the SNR: a single degenerate white dwarf, accreting wind material from a rather massive AGB star. The mass of the donor then implies that the white dwarf was also rather massive at the main sequence $\left(5-6 \mathrm{M}_{\odot}\right)$. Kepler's SNR provides therefore the best case that at least some Type Ia supernovae are caused by single degenerate white dwarf systems. 


\subsection{Problems with a single degenerate Type Ia scenario for SN 1604}

There two problems that need to be solved for the single degenerate scenario for SN 1604, or even for any Type Ia binary scenario:

1) The donor star must be relatively massive $\left(4-6 \mathrm{M}_{\odot}\right)$, and therefore a luminous star. A recent search by Kerzendorf et al (2014) did not reveal any bright enough star near the centre of Kepler's SNR. The former donor star is likely to still be bright, although, its outer envelope may have been removed by the supernova blast. Its space velocity should be close to that of the progenitor system. The lack of a donor star argues instead for a double degenerate explosion for SN 1604. A similar conclusion was drawn for other Type Ia SNRs, SNR 0509-675 and SN 1006 (Schaefer and Pagnotta, 2012; González Hernández et al, 2012), for which also no bright donor stars have been found. For Tycho's SNR a donor star has been identified (Ruiz-Lapuente, 2004), but this identification is disputed (Kerzendorf et al, 2013).

2) The velocity of the progenitor system must have been $\sim 250 \mathrm{~km} \mathrm{~s}^{-1}$. This implies that the system must have left the Galactic plane around 3 million year ago. Such a time scale is too short for any Type Ia scenario, which should have evolutionary time scales of $>50 \mathrm{Myr}$ (e.g. Claeys et al, 2014). One can of course speculate that Kepler's SNR is bound to the Galactic plane, and has been oscillating far up and below the Galactic plane for considerable longer times. This still creates, however, the problem of how to slingshot away a binary system with $250 \mathrm{~km} \mathrm{~s}^{-1}$. Runaway stars are usually created by close interactions of triple systems, but in $90 \%$ of the cases single stars are being ejected, and the highest velocities are not expected to be obtained by the ejected binaries (Leonard and Duncan, 1990). Moreover, in the AGB scenario for SN 1604 the binary system must have been relatively wide (Chiotellis et al, 2012). Nevertheless, observationally some high velocity binaries are known. A well known example is the Mira system, which has a space velocity of about $100 \mathrm{~km} \mathrm{~s}^{-1}$, and which consists also of an AGB star and a probable white dwarf (Martin et al, 2007).

Problem 1) is a problem for only the single degenerate scenario, but problem 2 ) is a problem for any Type Ia scenario involving a binary system.

\subsection{Was SN 1604 a core-degenerate Type Ia explosion?}

A possible solution to problem 1 is so-called core degenerate scenario (Ilkov and Soker, 2012; Tsebrenko and Soker, 2013) according to which a single degenerate system may form a common envelope at the end of the evolution of the secondary star (an AGB star). The white dwarf and the core of the AGB star merge at the very end of the AGB star evolution, or shortly thereafter. The resulting white dwarf may be super-Chandrasekhar, but gravitational collapse followed by an explosion can be delayed due to a rapid rotation of the massive white dwarf. Indeed, this scenario is somewhat intermediate between the single degenerate and the double degenerate scenario, as the supernova is clearly caused by a double degenerate merger, but 
the time scales involved can be similar to the single degenerate scenario. A consequence of this scenario is that the supernova mass does not have to be $1.38 \mathrm{M}_{\odot}$, but could be more. Another possible consequence for SN 1604 may be that there was high density material surrounding the progenitor, consisting of the envelope removed by the common envelope event. No light echo has yet been detected of SN 1604 (Rest et al, 2008), but if it will be detected and an optical spectrum can be obtained, perhaps we will learn more about the event itself and the presence of material in the immediate vicinity of the supernova.

\subsection{What can we learn from the historical light curve of SN 1604?}

Up to the 1990ies the hope was that the historical light curves of SN 1572 and SN 1604 could shed light on the maximum brightness of Type Ia supernovae, and hence be used to constrain the Hubble parameter (e.g. Danziger and Goss, 1980, Schaefer, 1996). Nowadays the Hubble parameter is known to sufficient detail, but the historical light curves remain of interest, as it may lead to sub-typing of the historical supernovae and provide estimates on the distance of SNRs (Section. 2). Baade (1943) was the first one to determine the historical light curve of SN 1604, and this was later augmented with magnitude estimates based on Korean observations by Clark and Stephenson (1977). These data have been compared to supernovae light curve by among others Schaefer (1996), and most recently Katsuda et al (2015).

The historical light curve of SN 1604 is shown in Fig. 6 with a typical magnitude error of 0.25-0.5. The historical light curve is compared to a number of recently observed Type Ia supernovae (Table 1 for an overview). These lightcurves have been scaled to the light curve of SN 1604 by taking into account the distance moduli $(\mu)$ and visual extinction parameters $\left(A_{V}\right)$ of the supernovae, and assuming a distance of $5 \mathrm{kpc}$ to $\mathrm{SN} 1604$ (somewhat in the middle of current estimates, Sect. (2) and $A_{V}=2.8$ for the historical supernova. The latter is based on the estimate of Blair et al (1991) for the non-radiative shock emission.

According to Type supernova light curve models, the peak magnitude of Type Ia supernovae is determined by the amount of radioactive ${ }^{56} \mathrm{Ni}$ produced in the explosion (typically $\sim 0.7 \mathrm{M}_{\odot}$ ), whereas the steepness of the decline after maximum is determined by the diffusion of heat generated by radioactivity to the photosphere of the supernova; the more mass the slower the decline (Arnett, 1979; Cappellaro et al, 1997; Dado and Dar, 2015). Hence, the low peak magnitude and fast decline of SN 1991bg has been attributed to a sub-Chandrasekhar Type Ia explosion (Turatto et al, 1996, Drout et al, 2013), whereas the very bright and slow declining SN 2009dc is thought to indicate a super-Chandrasekhar Type Ia (Silverman et al, 2011). The latter has a light curve that is even broader and brighter than SN 1991T, and its light curve has to be scaled by $\Delta V=0.5$ in order to provide even an approximate fit to SN 1604.

Recently Patnaude et al (2012) suggested that SN 1604 may have been similar to the bright Type Ia SN 1991T. For a distance of $5 \mathrm{kpc}$ the peak absolute peak 
magnitude of SN 1991T and SN 1604 match reasonably well, but SN 1991T has a much brighter late time light curve, and a slower post maximum decline. In order to make SN 1604 as luminous as SN 1991T at late times, the distance of SN 1604 has to be $\sim 7 \mathrm{kpc}$. So the light curve of SN 1991T does not seem to be an overall good template for SN 1604. The situation is even worse for the probable superChandrasekhar Type Ia SN,2009dc, which, even when scaled down in brightness, shows a profile that is too broad to fit the historical light curve.

Normal Type Ia supernova light curves (SN 1994D, SN 1996X, SN 2004eo) are in reasonable agreement with the historical light curve of SN 1604. In particular, they fit reasonably well around the peak and they agree reasonably well at very late times (300-400 days), but they are brighter than SN 1604 for days 100-200.

At the faint extreme, there are peculiar Type Ia supernovae such as SN $2009 \mathrm{dc}$, whose light curve has to shifted up by $\Delta V=1.5$ in Fig. 6 in order to have the peak brightness coincide with the peak of SN 1604. Such a shift would mean that SN 1604 would be at a distance of $2.5 \mathrm{kpc}$, which is lower than most independent distance estimates (Sect.2). As Katsuda et al (2015) showed, when properly scaled to match the peak magnitude of SN 1604, the light curve of SN 2009dc is rather similar to the light curve of SN 1604, as both are fastly declining in brightness after peak brightness. A similarly fast decline Type Ia was SN 2005ek, whose light curve was used to estimate the ejecta mass of $0.3-0.7 \mathrm{M}_{\odot}$, and a kinetic energy of $(2-5) \times 10^{50} \mathrm{erg}$. However, SN $2005 \mathrm{ek}$ was poor in ${ }^{56} \mathrm{Ni}$, at odds with what we know of SN 1604 (Sect.2.2). Perhaps one can still fit SN 1604 with a faint Type Ia model, if one allows for a small (sub-Chandrasekhar) progenitor mass, but with a large fraction of ${ }^{56} \mathrm{Ni}$. This would make a fainter supernova, with a rapid decline, but it could still allow for the high iron fraction in Kepler's SNR. The reason this may work is that in X-rays the fraction of iron can be much better determined than the absolute mass of iron. A consequence is then that the distance of Kepler's SNR is less than $5 \mathrm{kpc}$, as the supernova is fainter, but this can be accommodated with hydrodynamical models since the supernova is then probably also a sub-energetic Type Ia $\left(<10^{51} \mathrm{erg}\right.$, Chiotellis et al, 2012).

So what can we conclude from the historical light curve, with some reserve as the magnitudes derived from the historical observations have to be treated with some caution, and the extinction is uncertain? First of all, the light curves of normal Type Ia seem overall in better agreement with the light curve of SN 1604 than the light curve of SN 1991T. Secondly, the light curve of SN 1604 may have declined rather rapidly after peak brightness. The evidence for this is uncertain, but could indicate a sub-Chandrasekhar explosion. However, in order to account for the high iron fraction of Kepler's SNR, SN 1991bg and SN 2005ek are probably not the right models. Moreover, a sub-Chandrasekhar model, would result in a shorter distance of Kepler's SNR.

A possibility that needs to be explored better is whether it is possible that the fast decline of SN 1604 could perhaps be the result of an overall faster expansion, and/or a distribution of nickel closer to the photosphere. This helps the heat generated by ${ }^{56} \mathrm{Ni}$ to diffuse faster to the photosphere, resulting in a more rapid 
Table 1: A comparison of SN 1604 to the Type Ia SNe shown in Fig. 6.

\begin{tabular}{lccccll|}
\hline \hline & $V_{\max }$ & $A_{V}$ & $m-M$ & $M_{V}$ & Remark & References \\
\hline SN 1604: & & & & & \\
@ 3 kpc & -2.75 & 2.8 & 12.39 & -17.94 & & Blair et al (1991) \\
& -2.75 & 3.5 & 12.39 & -18.64 & & Danziger and Goss (1980) \\
@ 6 kpc & -2.75 & 2.8 & 13.89 & -19.44 & & Blair et al (1991) \\
& -2.75 & 3.5 & 13.89 & -20.14 & & Danziger and Goss (1980) \\
\hline SN 1991T & 11.50 & 0.37 & 30.57 & -19.44 & Bright Type Ia & Sasdelli et al (2014) \\
SN 1991bg & 13.96 & 0.12 & 31.61 & -17.77 & Sub-Chandrasekhar & Turatto et al (1996); Gómez and Richtler (2004) \\
SN 1994D & 11.90 & 0.08 & 30.82 & -19.00 & Normal Type Ia & Cappellaro et al (1997); Feldmeier et al (2007) \\
SN 1996X & 13.21 & 0.22 & 31.15 & -18.16 & Normal Type Ia & Salvo et al (2001) \\
SN 2004eo & 15.35 & 0.34 & 34.12 & -19.11 & Sub-Chandrasekhar? & Pastorello et al (2007) \\
SN 2009dc & 15.35 & 0.31 & 34.93 & -19.89 & Super-Chandrasekhar & Silverman et al 2011) \\
\hline
\end{tabular}

post-maximum decline. In any case, the fast decline is inconsistent with a superChandrasekhar explosion. This is of interest given the suggestion that SN 1604 could provide a evidence for the core-degenerate model (Sect. 4.3 Tsebrenko and Soker, 2013), which can result in super-Chandrasekhar Type Ia explosions.

\section{Conclusions}

The remnant of SN 1604, Kepler's SNR, is one of the most remarkable SNRs, with its high bulk velocity of $250 \mathrm{~km} \mathrm{~s}^{-1}$. SN 1604 is now generally regarded to have been a Type Ia supernova, whose remnant is interacting with wind material from its progenitor system. One can even infer that the donor star must have been a 4-6 $\mathrm{M}_{\odot}$ star that had evolved to the AGB phase. But this single degenerate Type Ia scenario for SN 1604 has only one problem: a surviving donor has not been detected. A core-degenerate supernova scenario, may offer a viable alternative theory, but it has its own problems. Whatever the explosion scenario, the high space velocity of the progenitor system remains mysterious, as it requires the ejection of a binary system out of the Galactic plane with $\sim 250 \mathrm{~km} \mathrm{~s}^{-1}$.

In Johannes Kepler's age SN 1604 was recognised as a unique, mysterious event that was thought to have profound implications for mankind. We may no longer think that SN 1604 is of prime importance for mankind, but SN 1604 is of prime importance for understanding Type Ia supernovae. SN 1604 has, even more than $400 \mathrm{yr}$ after discovery, still profound implications for understanding cosmic phenomena, and it has still not shared all its mysteries with us.

\section{References}

Aharonian F, Akhperjanian AG, Barres de Almeida U, Bazer-Bachi AR., Behera B., Beilicke M., et al (2008) HESS upper limits for Kepler's supernova remnant. A\&A488:219-223, DOI 10.1051/0004-6361:200809401,0806.3347 


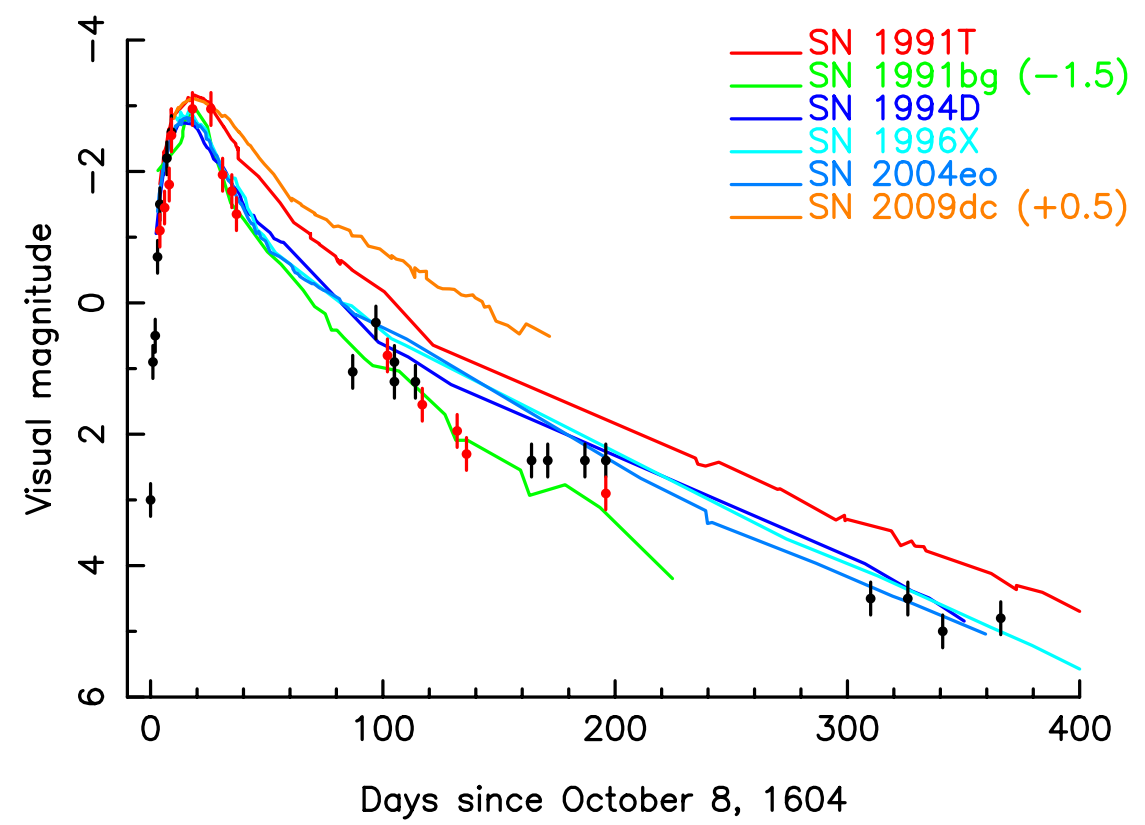

Figure 6: The light curve of SN 1604 based on the European observations as interpreted by Baade (1943, black data points) and the Korean observations collected by Stephenson and Green (2002, red data points). For comparison the light curves of several other Type Ia supernovae have been over-plotted. These curves have been scaled to that of SN 1604, assuming a distance for SN 1604 of $5 \mathrm{kpc}$ and $A_{V}=2.8$. More or less normal Type Ia supernovae light curves have been assigned bluish colours. See Table 1 for details. 
Arnett WD (1979) On the theory of Type I supernovae. ApJ230:L37-L40, DOI $10.1086 / 182957$

Baade W (1943) Nova Ophiuchi of 1604 AS a Supernova. ApJ97:119

Bandiera R (1987) The origin of Kepler's supernova remnant. ApJ319:885-892, DOI 10.1086/165505

Bandiera R, van den Bergh S (1991) Changes in the optical remnant of Kepler's supernova during the period 1942-1989. ApJ374:186-201

Blair WP, Long KS, Vancura O (1991) A detailed optical study of Kepler's supernova remnant. ApJ366:484-494,

Blair WP, Ghavamian P, Long KS, Williams BJ, Borkowski KJ, Reynolds SP, Sankrit R (2007) Spitzer Space Telescope Observations of Kepler's Supernova Remnant: A Detailed Look at the Circumstellar Dust Component. ApJ662:9981013, DOI 10.1086/518414, arXiv:astro-ph/0703660

Borkowski KJ, Sarazin CL, Blondin JM (1994) On the X-ray spectrum of Kepler's supernova remnant. ApJ429:710-725

Braun R (1987) The structure and dynamics of young supernova remnants - New constraints from observations of shock-heated dust. A\&A171:233-251

Burkey MT, Reynolds SP, Borkowski KJ, Blondin JM (2013) X-Ray Emission from Strongly Asymmetric Circumstellar Material in the Remnant of Kepler's Supernova. ApJ764:63, DOI 10.1088/0004-637X/764/1/63, 1212.4534

Cappellaro E, Mazzali PA, Benetti S, Danziger IJ, Turatto M, della Valle M, Patat F (1997) SN IA light curves and radioactive decay. A\&A328:203-210, astro-ph/9707016

Cassam-Chenaï G, Decourchelle A, Ballet J, Hwang U, Hughes JP, Petre R, et al (2004) XMM-Newton observation of Kepler's supernova remnant. A\&A414:545-558, DOI 10.1051/0004-6361:20031551, astro-ph/ 0310687

Chevalier RA (1982) Self-similar solutions for the interaction of stellar ejecta with an external medium. ApJ258:790-797

Chiotellis A, Schure KM, Vink J (2012) The imprint of a symbiotic binary progenitor on the properties of Kepler's supernova remnant. A\&A537:A139, DOI 10.1051/0004-6361/201014754, 1103.5487

Claeys JSW, Pols OR, Izzard RG, Vink J, Verbunt FWM (2014) Theoretical uncertainties of the Type Ia supernova rate. A\&A563:A83, DOI 10.1051/0004-6361/ 201322714, 1401.2895

Clark DH, Stephenson FR (1977) The historical supernovae. Pergamon Press

Dado S, Dar A (2015) Analytical Expressions for Light Curves of Ordinary and Superluminous Type Ia Supernovae. ApJ809:32, DOI 10.1088/0004-637X/809/ $1 / 32$

Danziger IJ, Goss WM (1980) The distance of Kepler's supernova remnant. MNRAS190:47P-49P

Delaney T, Rudnick L (2003) The first measurement of Cassiopeia A's forward shock expansion rate. ApJ589:818

Dennefeld M (1982) A spectrophotometric study of Kepler supernova remnant. 
A\&A112:215-222

Dickel JR, Sault R, Arendt RG, Korista KT, Matsui Y (1988) The evolution of the radio emission from Kepler's Supernova remnant. ApJ330:254-263

Douvion T, Lagage PO, Cesarsky CJ, Dwek E (2001) Dust in the Tycho, Kepler and Crab supernova remnants. A\&A373:281-291

Draine BT (1981) Infrared emission from dust in shocked gas. ApJ245:880-890, DOI $10.1086 / 158864$

Drout MR, et al (2013) The Fast and Furious Decay of the Peculiar Type Ic Supernova 2005ek. ApJ774:58, DOI 10.1088/0004-637X/774/1/58, 1306.2337

Dwek E (1987) The infrared diagnostic of a dusty plasma with applications to supernova remnants. ApJ322:812-821, DOI 10.1086/165774

Feldmeier JJ, Jacoby GH, Phillips MM (2007) Calibrating Type Ia Supernovae Using the Planetary Nebula Luminosity Function. I. Initial Results. ApJ657:7694, DOI 10.1086/510897, astro-ph/0611231

Fesen RA, Becker RH, Blair WP (1987) Discovery of fast-moving nitrogen-rich ejecta in the supernova remnant Cassiopeia A. ApJ313:378-388

Gomez HL, Dunne L, Ivison RJ, Reynoso EM, Thompson MA, Sibthorpe B, et al (2009) Accounting for the foreground contribution to the dust emission towards Kepler's supernova remnant. MNRAS397:1621-1632, DOI 10.1111/j. 1365-2966.2009.15061.x, 0905.2564

Gomez HL, Clark CJR, Nozawa T, Krause O, Gomez EL, Matsuura M, et al (2012) Dust in historical Galactic Type Ia supernova remnants with Herschel. MNRAS420:3557-3573, DOI 10.1111/j.1365-2966.2011.20272.x, 1111.6627

Gómez M, Richtler T (2004) The globular cluster system of NGC 4374. A\&A415:499-508, DOI 10.1051/0004-6361:20034610, astro-ph/ 0311188

González Hernández JI, Ruiz-Lapuente P, Tabernero HM, Montes D, Canal R, Méndez J, Bedin LR (2012) No surviving evolved companions of the progenitor of SN 1006. Nature489:533-536, DOI 10.1038/nature11447, 1210.1948

Granada MA (2005) The Discussion between Kepler and Roeslin on the Nova of 1604. In: Turatto M, Benetti S, Zampieri L, Shea W (eds) 1604-2004: Supernovae as Cosmological Lighthouses, Astronomical Society of the Pacific Conference Series, vol 342, p 30

Green DA, Reynolds SP, Borkowski KJ, Hwang U, Harrus I, Petre R (2008) The radio expansion and brightening of the very young supernova remnant G1.9+0.3. MNRAS387:L54-L58, DOI 10.1111/j.1745-3933.2008.00484.x, 0804.2317

Helder EA, Vink J, Bykov AM, Ohira Y, Raymond JC, Terrier R (2012) Observational Signatures of Particle Acceleration in Supernova Remnants. Space Science Rev.173:369-431, DOI 10.1007/s11214-012-9919-8, 1206.1593

Hwang U, Gotthelf EV (1997) X-Ray Emission-Line Imaging and Spectroscopy of Tycho's Supernova Remnant. ApJ475:665

Ilkov M, Soker N (2012) Type Ia supernovae from very long delayed explosion of core-white dwarf merger. MNRAS419:1695-1700, DOI 10.1111/j.1365-2966. 
2011.19833.x, 1106.2027

Karakas AI (2010) Updated stellar yields from asymptotic giant branch models. MNRAS403:1413-1425, DOI 10.1111/j.1365-2966.2009.16198.x, 0912 . 2142

Katsuda S, Tsunemi H, Uchida H, Kimura M (2008) Forward Shock Proper Motions of Kepler's Supernova Remnant. ApJ689:225-230, DOI 10.1086/592376, 0812.0339

Katsuda S, Mori K, Maeda K, Tanaka M, Koyama K, Tsunemi H, Nakajima H, Maeda Y, Ozaki M, Petre R (2015) Kepler's Supernova: An Overluminous Type Ia Event Interacting with a Massive Circumstellar Medium at a Very Late Phase. ApJ808:49, DOI 10.1088/0004-637X/808/1/49, 1506.03135

Kerzendorf WE, Yong D, Schmidt BP, Simon JD, Jeffery CS, Anderson J, et al (2013) A High-resolution Spectroscopic Search for the Remaining Donor for Tycho's Supernova. ApJ774:99, DOI 10.1088/0004-637X/774/2/99, 1210.2713

Kerzendorf WE, Childress M, Scharwächter J, Do T, Schmidt BP (2014) A Reconnaissance of the Possible Donor Stars to the Kepler Supernova. ApJ782:27, DOI 10.1088/0004-637X/782/1/27, 1309.5964

Kinugasa K, Tsunemi H (1999) ASCA Observation of Kepler's Supernova Remnant. PASJ51:239-252

Kosenko D, Helder EA, Vink J (2010) The kinematics and chemical stratification of the type Ia supernova remnant 0519-69.0. An XMM-Newton and Chandra study. A\&A519:A11+, DOI 10.1051/0004-6361/200913903, 1001.0983

Lagage PO, Claret A, Ballet J, Boulanger F, Cesarsky CJ, Cesarsky D, Fransson C, Pollock A (1996) Dust formation in the Cassiopeia A supernova. A\&A315:L273-L276

Leibowitz EM, Danziger IJ (1983) Spectrophotometry in the galactic supernova remnants RCW 86, 103 and Kepler. MNRAS204:273-287

Leonard PJT, Duncan MJ (1990) Runaway stars from young star clusters containing initial binaries. II - A mass spectrum and a binary energy spectrum. AJ99:608-616, DOI 10.1086/115354

Maoz D, Mannucci F, Nelemans G (2014) Observational Clues to the Progenitors of Type Ia Supernovae. Ann. Rev. A\&A52:107-170, DOI 10.1146/ annurev-astro-082812-141031, 1312.0628

Martin DC, et al (2007) A turbulent wake as a tracer of 30,000years of Mira's mass loss history. Nature448:780-783, DOI 10.1038/nature06003

McSaveney JA, Wood PR, Scholz M, Lattanzio JC, Hinkle KH (2007) Abundances in intermediate-mass AGB stars undergoing third dredge-up and hot-bottom burning. MNRAS378:1089-1100, DOI 10.1111/j.1365-2966.2007. 11845.x, 0704.1907

Minkowski R (1959) Optical observations of nonthermal galactic radio sources. In: Bracewell RN (ed) URSI Symp. 1: Paris Symposium on Radio Astronomy, IAU Symposium, vol 9, p 315

Morgan HL, Dunne L, Eales SA, Ivison RJ, Edmunds MG (2003) Cold Dust in Kepler's Supernova Remnant. ApJ597:L33-L36, DOI 10.1086/379639, 
astro-ph/0309233

Park S, Badenes C, Mori K, Kaida R, Bravo E, Schenck A, et al (2013) A Supersolar Metallicity for the Progenitor of Kepler's Supernova. ApJ767:L10, DOI 10.1088/2041-8205/767/1/L10, 1302.5435

Pastorello A, et al (2007) ESC and KAIT observations of the transitional Type Ia SN 2004eo. MNRAS377:1531-1552, DOI 10.1111/j.1365-2966.2007.11700.x, astro-ph/0702565

Patnaude DJ, Badenes C, Park S, Laming JM (2012) The Origin of Kepler's Supernova Remnant. ApJ756:6, DOI 10.1088/0004-637X/756/1/6, 1206.6799

Rest A, et al (2008) Spectral Identification of an Ancient Supernova Using Light Echoes in the Large Magellanic Cloud. ApJ680:1137-1148, DOI 10.1086/ 587158, 0801.4762

Reynolds SP, Borkowski KJ, Hwang U, Hughes JP, Badenes C, Laming JM, Blondin JM (2007) A Deep Chandra Observation of Kepler's Supernova Remnant: A Type Ia Event with Circumstellar Interaction. ApJ668:L135-L138, DOI 10.1086/522830, arXiv:0708.3858

Reynoso EM, Goss WM (1999) A New Determination of the Distance to Kepler's Supernova Remnant. AJ118:926-929

Ruiz-Lapuente P (2004) Tycho Brahe's Supernova: Light from Centuries Past. ApJ612:357-363

Salvo ME, Cappellaro E, Mazzali PA, Benetti S, Danziger IJ, Patat F, Turatto M (2001) The template type Ia supernova 1996X. MNRAS321:254-268, arXiv: astro-ph/0009065

Sankrit R, Blair WP, Delaney T, Rudnick L, Harrus IM, Ennis JA (2005) HST/ACS imaging of a Balmer-dominated shock in Kepler's supernova remnant. Advances in Space Research 35:1027-1030, DOI 10.1016/j.asr.2004.11.018

Sankrit R, Blair WP, Frattare LM, Rudnick L, DeLaney T, Harrus IM, Ennis JA (2008) Hubble Space Telescope/Advanced Camera for Surveys Narrowband Imaging of the Kepler Supernova Remnant. AJ135:538-547, DOI 10.1088/0004-6256/135/2/538

Sasdelli M, Mazzali PA, Pian E, Nomoto K, Hachinger S, Cappellaro E, Benetti S (2014) Abundance stratification in Type Ia supernovae - IV. The luminous, peculiar SN 1991T. MNRAS445:711-725, DOI 10.1093/mnras/stu1777, 1409.0116

Schaefer BE (1996) Peak Brightnesses of Historical Supernovae and the Hubble Constant. ApJ459:438, DOI 10.1086/176906

Schaefer BE, Pagnotta A (2012) An absence of ex-companion stars in the type Ia supernova remnant SNR 0509-67.5. Nature481:164-166, DOI 10.1038/ nature 10692

Silverman JM, Ganeshalingam M, Li W, Filippenko AV, Miller AA, Poznanski D (2011) Fourteen months of observations of the possible superChandrasekhar mass Type Ia Supernova 2009dc. MNRAS410:585-611, DOI 10.1111/j.1365-2966.2010.17474.x, 1003.2417

Sollerman J, Ghavamian P, Lundqvist P, Smith RC (2003) High resolution spec- 
troscopy of Balmer-dominated shocks in the RCW 86, Kepler and SN 1006 supernova remnants. A\&A407:249-257

Stephenson FR, Green DA (2002) Historical supernovae and their remnants. Oxford: Clarendon Press

Toledo-Roy JC, Esquivel A, Velázquez PF, Reynoso EM (2014) A 3D numerical model for Kepler's supernova remnant. MNRAS442:229-238, DOI 10.1093/ mnras/stu 880

Tsebrenko D, Soker N (2013) Type Ia supernovae inside planetary nebulae: shaping by jets. MNRAS435:320-328, DOI 10.1093/mnras/stt1301, 1305.1845

Turatto M, Benetti S, Cappellaro E, Danziger IJ, Della Valle M, Gouiffes C, Mazzali PA, Patat F (1996) The properties of the peculiar type Ia supernova 1991bg. I. Analysis and discussion of two years of observations. MNRAS283:1-17, astro-ph/9605178

van Adelsberg M, Heng K, McCray R, Raymond JC (2008) Spatial Structure and Collisionless Electron Heating in Balmer-dominated Shocks. ApJ689:10891104, DOI 10.1086/592680, 0803.2521

van den Bergh S, Kamper KW (1977) The remnant of Kepler's supernova. ApJ218:617-619, DOI 10.1086/155719

van den Bergh S, Marscher AP, Terzian Y (1973) An Optical Atlas of Galactic Supernova Remnants. ApJS26:19, DOI 10.1086/190278

Vink J (2008b) The Kinematics of Kepler's Supernova Remnant as Revealed by Chandra. ApJ689:231-241, DOI 10.1086/5923750803.4011

Vink J, Yamazaki R, Helder EA, Schure KM (2010) The Relation Between Postshock Temperature, Cosmic-ray Pressure, and Cosmic-ray Escape for Nonrelativistic Shocks. ApJ722:1727-1734, DOI 10.1088/0004-637X/722/2/1727, 1008.4367

Williams BJ, Borkowski KJ, Reynolds SP, Ghavamian P, Blair WP, Long KS, Sankrit R (2012) Dust in a Type Ia Supernova Progenitor: Spitzer Spectroscopy of Kepler's Supernova Remnant. ApJ755:3, DOI 10.1088/0004-637X/755/1/3, 1206.1054

Woosley SE, Kasen D, Blinnikov S, Sorokina E (2007) Type Ia Supernova Light Curves. ApJ662:487-503, DOI 10.1086/513732, arXiv:astro-ph/ 0609562

\section{acknowledgement}

This chapter is born out of a long fascination for Kepler's supernova remnant; and my view on the supernova remnant was influenced by my collaboration with my former graduate students Alexandros Chiotellis and Klara Schure. During this collaboration also discussions with Onno Pols about the evolution of binary stars and the process of hot bottom burning were much appreciated. 